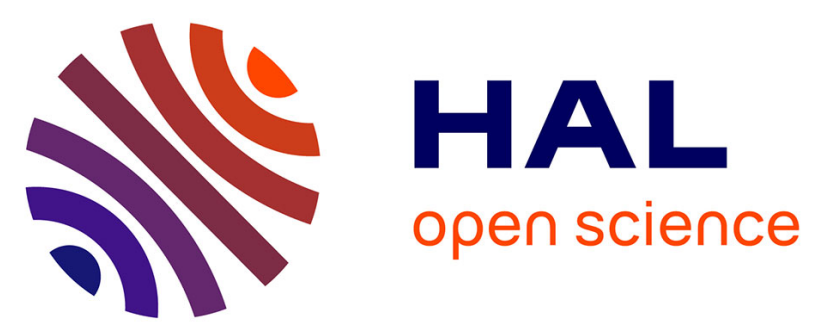

\title{
Labelling IL-18 with alkaloids: towards the use of cytokines as carrier molecules in chemotherapy
}

Fracisco J Martin-Martinez, Julia Contreras-García, Horacio Pérez-Sánchez, José P Cerón-Carrasco

\section{- To cite this version:}

Fracisco J Martin-Martinez, Julia Contreras-García, Horacio Pérez-Sánchez, José P Cerón-Carrasco. Labelling IL-18 with alkaloids: towards the use of cytokines as carrier molecules in chemotherapy. Theoretical Chemistry Accounts: Theory, Computation, and Modeling, 2019, 138 (8), 10.1007/s00214019-2483-5 . hal-02407860

\section{HAL Id: hal-02407860 \\ https://hal.sorbonne-universite.fr/hal-02407860}

Submitted on 12 Dec 2019

HAL is a multi-disciplinary open access archive for the deposit and dissemination of scientific research documents, whether they are published or not. The documents may come from teaching and research institutions in France or abroad, or from public or private research centers.
L'archive ouverte pluridisciplinaire HAL, est destinée au dépôt et à la diffusion de documents scientifiques de niveau recherche, publiés ou non, émanant des établissements d'enseignement et de recherche français ou étrangers, des laboratoires publics ou privés. 


\title{
Labelling IL-18 with alkaloids: towards the use of cytokines as carrier molecules in chemotherapy
}

\author{
Fracisco J. Martín-Martínez ${ }^{\mathrm{a}}$, Julia Contreras-García ${ }^{\mathrm{b}}$, Horacio \\ Pérez-Sánchez ${ }^{\mathrm{c}}$, José P. Cerón-Carrasco ${ }^{\mathrm{c}, *}$ \\ ${ }^{a}$ Department of Civil and Environmental Engineering, Massachusetts Institute of \\ Technology (MIT), USA \\ ${ }^{b}$ UPMC Université Paris 06, UMR 7616 CNRS. Laboratoire de Chimie Theorique, Case \\ Courrier 137, 4 Place Jussieu, F-75005, Paris, France \\ ${ }^{c}$ Bioinformatics and High Performance Computing Research Group (BIO-HPC), \\ Computer Engineering Department. Universidad Católica San Antonio de Murcia \\ (UCAM). Campus de los Jerónimos, 3010\%, Murcia, Spain
}

\begin{abstract}
Recent in vivo models demonstrated that immune checkpoint cancer therapy can be improved by injecting interleukine (IL)-18, a proinflammatory cytokine of the IL-1 family. Aiming to enhance the beneficial action of citokines in cancer treatments, we used here docking, non-covalent interaction analysis and molecular dynamics simulation to determine their ability for embedding alkaloid-based drugs. According to our simulations, three alkaloids with anticancer activity, e.g., paclitaxel, vincristine and vinorelbine, are efficiently retained in the central cavity of IL-18. The reported results pave a new synthetic route for designing novel bifunctional carrier-cargo systems with enhanced anticancer activity.
\end{abstract}

Keywords: immune checkpoint blocking therapy, cancer, interleukin, docking, molecular dynamics, non-covalent interactions

\section{Introduction}

$2 \quad$ More than ten years ago, Cragg and Newman published their seminal 3 paper "Plants as a source of anti-cancer agent", an updated review over 4 the applications of the traditional medicine within the framework of current

*jpceron@ucam.edu 
clinical therapies [1]. As discussed by these authors, the use of plant-derived extracts as anticancer agents has attracted an increasing attention with a particular focus on alkaloids, which are known exhort a strong anticancer activity [2]. Indeed, alkaloid-based drugs has been proposed for the treatment of several disorders including carcinomas, lung, prostate and breast cancer [3]. Although the biological mechanism of alkaloids is rather than complex at the cellular level [4], the inhibition of the microtubulin function is now accepted to be the key step in the their anticancer activity [5].Unfortunately, that reaction is not site-specific and alkaloids show severe side effects due to their high cytotoxic in healthy cells.

To circumvent chemotherapy risks, novel drugs can be encapsulated into a carrier molecule [6], that is, the formation of a host-guest complex that avoid an early activation of embedded drug while simultaneously concentrating their action in the tumor area [7]. Such approach is also possible for alkaloids. As Cragg and Newman stated, "the ability to attach agents to carrier molecules directed to specific tumors, shows promise for effectively targeting highly cytotoxic natural products to the tumors while avoiding their toxic side effects on normal tissues" [1]. In the same vein, an attractive idea was recently proposed by Yadav an co-workers, who used theoretical simulations to determine the ability of trastuzumab (Herceptin $\left.{ }^{\circledR}\right)$ to embed three alkaloids: paclitaxel (Taxol ${ }^{\circledR}$ ), vincristine (Oncovin ${ }^{\circledR}$ ) and vinorelbine (Navelbine $\left.{ }^{\circledR}\right)$ [8]. Trastuzumab is a humanized monoclonal antibody that selectively binds to the HER2/neu over-expressed protein in breast cancer patients $[9,10]$. In this case, both carrier and cargo molecules posses anticancer activity, so that they can be defined as bifunctional drugs [11-14].

Computational simulations can be used to broaden the chemical space of carrier molecules beyond traditional antibodies. Here we aim to assess the use of interleukin (IL)-18 as carrier molecule. IL-18 is a proinflammatory cytokine of the IL-1 family that improves immune checkpoint blocking cancer therapy [15]. Although its biological mechanism of action is still under debate, the beneficial effects of IL-18 might be associated to the activation of cytotoxic pre-mNK cells, low accumulation of regulatory $\mathrm{T}$ cells, and suppression of soluble inhibitor secretion of IL-10 and TGF $\beta$ [15]. The present contribution provides the first comprehensive insights on the use of this cytokine as carrier material for paclitaxel, vincristine and vinorelbine anticancer drugs. More specifically, blind docking (BD), molecular dynamics (MD) simulations and non-covalent interaction (NCI) analyses are carried out to assess the distribution of potential binding sites across IL-18 protein 
surface and the stability of the IL-18 embedded alkaloids. Our theoretical predictions can help to improve immune checkpoint cancer immunotherapy based on novel IL-18 bifunctional carrier-cargo systems.

\section{Computational Details}

The initial model for the host system was built up by using the crystal structure recently resolved by Tsutsumi and co-workers, which was deposited at Protein Data Bank (PDB) [16] with code 1WO2 [17]. Hydrogen atoms were added and and cap termini were included with the Protein Preparation Wizard module implemented in Maestro [18]. Protonation states of all side chains were defined at $\mathrm{pH}=7$ using PROPKA 3.1 [19]. The three selected alkaloids (paclitaxel, vincristine and vinorelbine) were subsequently docked into the refined IL-18 structure using a BD approach [20], where multiple parallel and independent docking simulation were performed around geometric centers of all residues. BD simulations provide first insights into the ligand interaction pattern with IL-18, namely, which residues of the protein (not restricted to a particular cavity, but considering the whole protein surface) are involved, and how these interactions contribution to the total protein-ligand interaction energy [21]. All individual docking calculations in the BD approach were carried out with AutoDock Vina [22]. As discussed elsewhere [22], the scoring function implemented in composed by two electrostatic terms (specified as gauss1 and gauss2), and repulsive, hydrophobic, hydrogen bonds (H-bonds) and an entropic term linearly dependent on the number of rotatable bonds of the ligand.

The top BD pose of each alkaloid-based drug, namely, the one with the highest value of the scoring function, can be indentified as the most favourable binding site and was retained for the further analysis/simulations. To this end, NCIPlot code was applied to identify the nature of the chemical interactions established between the drug and the carrier molecule [23, 24]. That scheme provides a qualitative description of the main chemical contacts present in a host-guess system by combining the electron density $\rho$ over all atoms and the associated reduced gradient $\nabla$ as follows:

$$
s=\frac{1}{2\left(3 \pi^{2}\right)^{1 / 3}} \frac{|\nabla \rho|}{\rho^{4 / 3}}
$$

which enables to visualize weak interactions as colored isosurfaces of the reduced density gradient, $s[25]$. 
The resulting IL-18-embedded drug model systems were eventually located in a orthorhombic water box by defining a buffer distance of $10 \AA$ in $\mathrm{X}, \mathrm{Y}$ and $\mathrm{Z}$ directions by using the simple point charge (SPC) model [26]. Sodium cations were added to counterbalance the total electric charge. Additional sodium and chloride ions were added to reproduce the physiological $\mathrm{NaCl}$ salt concentration of $0.15 \mathrm{M}$. The whole solvated host-guest systems were intially minimizated for 2000 steps with the steepest descent scheme by imposing a convergence threshold of $1.0 \mathrm{kcal} / \mathrm{mol} / \AA$. The solvated systems were next relaxed with a multi-step protocol that includes a solute-restrained minimization, free-restrain minimization, NVT simulation of $24 \mathrm{ps}$ at $\mathrm{T}=10$ $\mathrm{K}$, NPT simulations at $\mathrm{T}=10 \mathrm{~K}$ and $\mathrm{P}=1 \mathrm{~atm}$. For the production phase, the temperature was set to $300 \mathrm{~K}$ with the Nosé-Hoover algorithm with a relaxation time of $1.0 \mathrm{ps}[27,28]$. Pressure was set at 1 bar using the Martyna-Tobias-Klein barostat with an isotropic coupling and a relaxation time of $2.0 \mathrm{ps}$ [29]. The RESPA integrator was used to integrate equations of motions with a $2.0 \mathrm{fs}$ time step for bonded and close interactions and a 6.0 fs time step for farther interactions [30]. A cutoff of $9 \AA$ was imposed to all non-bonded interactions. Van der Waals interactions were assesing using a cut-off radius of $9 \AA$ and the electrostatic part was defined using the Particle Mesh Ewald (PME) [31] method with a tolerance limit of $10^{-9}$. All MD simulations were carried out with the OPLS-2005 force field as implemented in Desmond code [32].

\section{Results and discussion}

Results obtained from BD simulations are summarized in Figures 1 and 2 , which provides the location and energetics of the different poses clusters across the IL-18 surface. According to the visual inspection of the best poses, the three selected alkaloids fit into the central region of the IL-18 structure. The histograms included in the left panel show the distribution of pose clusters. Pink bars represent the number of poses whose scoring function value falls into the assigned interval. Blue bars are specified as follows; the pose with the highest value of the scoring function is assigned a blue bar. Next, the clash of poses with lower values of the scoring function is calculated against the top pose. In case there is collision, pink color is assigned, otherwise it means a new cluster of poses is found and a blue bar is designated. This process is repeated until no more poses can be processed and afterwards, all pose clusters have been located. 


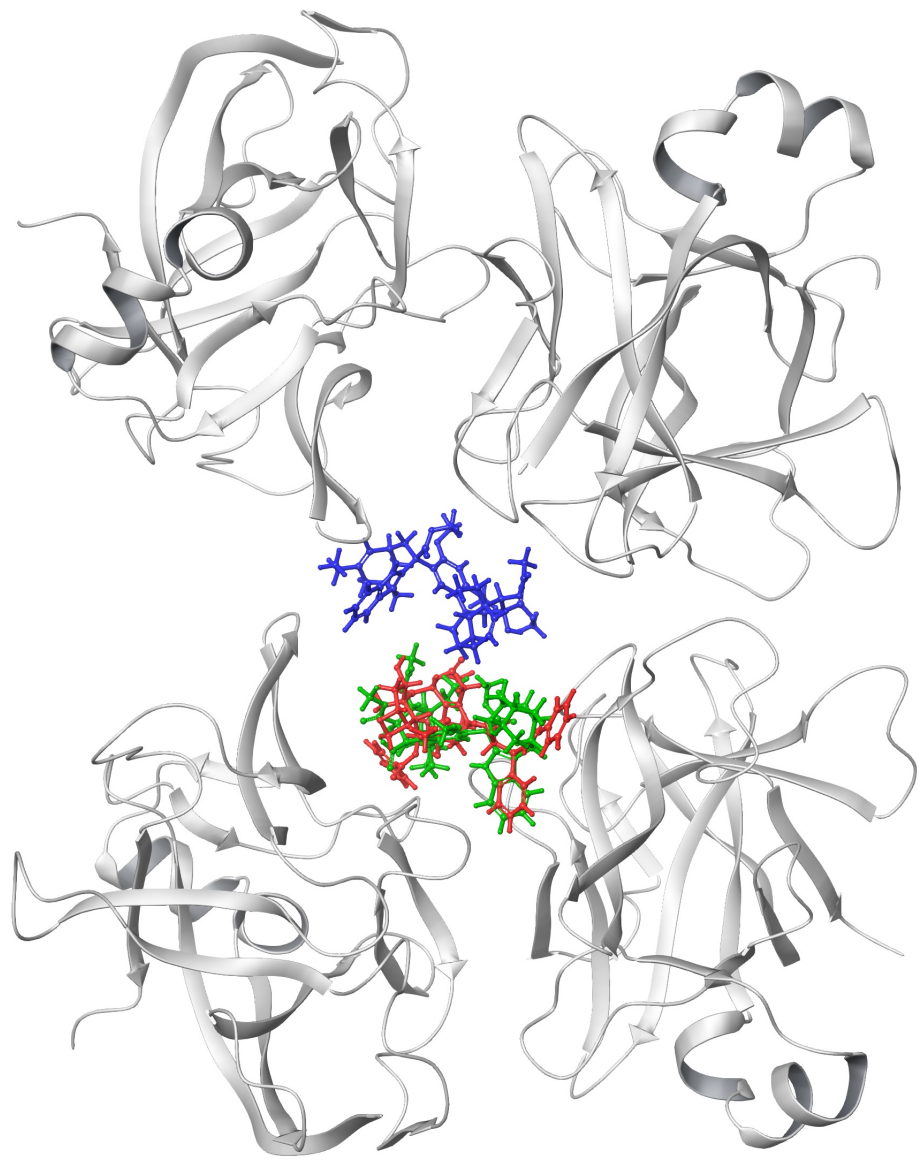

Figure 1: Conformations of the top docked poses. Ligands are shown as sticks: paclitaxel in red, vincristine in gren and vinorelbine in blue. The structure of the IL-18 cytokine is displayed as gray cartoon. 
That outcomes hints that the cytokine is preferentially loaded into its core structure, e.g., without altering its more external chemical region, the biological activity is consequently preserved. consequently act as an efficient carrier for any of these alkaloids. As one can see in Figure 2, the computed top poses lie to the left end of the histogram with high scoring function values $(<-7 \mathrm{kcal} / \mathrm{mol})$, which indicate a large affinity of the ligands. Figure 2 also reveals that several pose clusters (blue bars) are found close to the top pose. In all cases there are no big differences, regarding scoring function values, between clusters, which means there is no clear binding site preference for the compounds. The number of clusters goes around 20 , so the protein would in principle be able to transport more than 10 molecules at the same time. Figure 2 also depicts the energetic analyses of the previously mentioned top poses. We can see that main stabilizing interactions in all cases are related with hydrogen bonds and favorable hydrophobic contacts. These theoretical findings are relevant for our study since they suggest or confirm the utility of this protein for carrier purposes.

Docking provides a cost-effective evaluation of the protein domain(s) in interaction with drugs, a prerequisite for assessing all possible binding pockets [33]. However, further calculations are necessary to fully understand the biological mechanism of action. A NCI analysis is performed to provide a qualitative picture of the drug binding modes of the top BD poses and, therefore, complete the results arising from docking. The NCI analysis is summarized in Figure 3. In the selected color scheme the H-bonds are shown as blue surfaces and weak attractive interactions are given as green regions. Let us start with the paclitaxel drug. According to the computed surfaces, large attractive interactions are established between this drug and IL-18 through H-bonding with D:Ile80 and D:Lys135. This non-covalent interactions are highlighted by blue surfaces in left panel of Figure 3. Additional dispersion interactions appear on the benzenic moiety, that partially contribute to fix paclitaxel in the binding site. For instance, one can observe a weak attraction with the lateral chain of redisue D:Ile137. Additional interactions with D:Ile81 can be also identified in the binding site. It is also remarkable the interaction with B:Lys79. Paclitaxel is located very close to the latter residue so that an steric clash appears, which is identified by red surface in the NCI analysis. It is worth noting here that the major drawbacks of docking protocols are the lack of protein flexibility (it is usually kept as a rigid entity upon drug binding), which may lead to unreliable protein-drug contacts. Middel panel of Figure 3 illustrates the interactions established between vin- 
paclitaxel
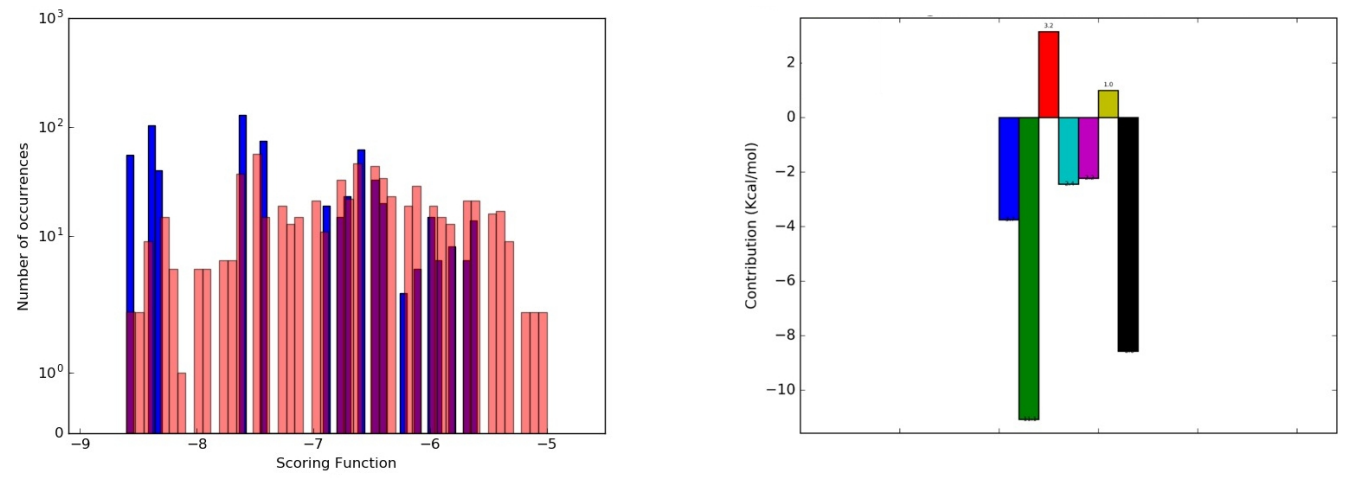

vincristine
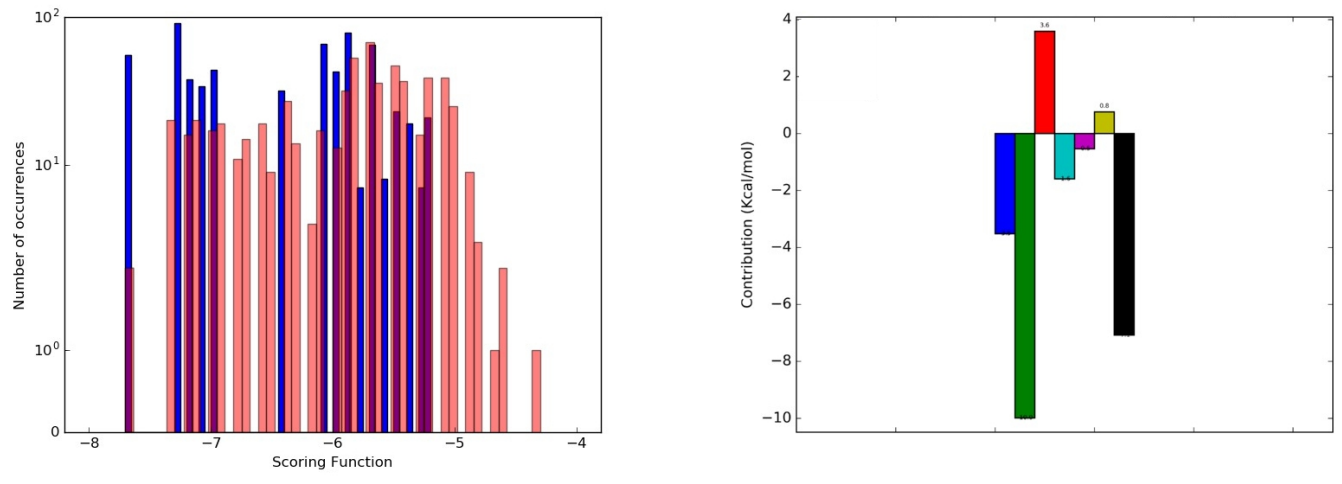

vinorelbine
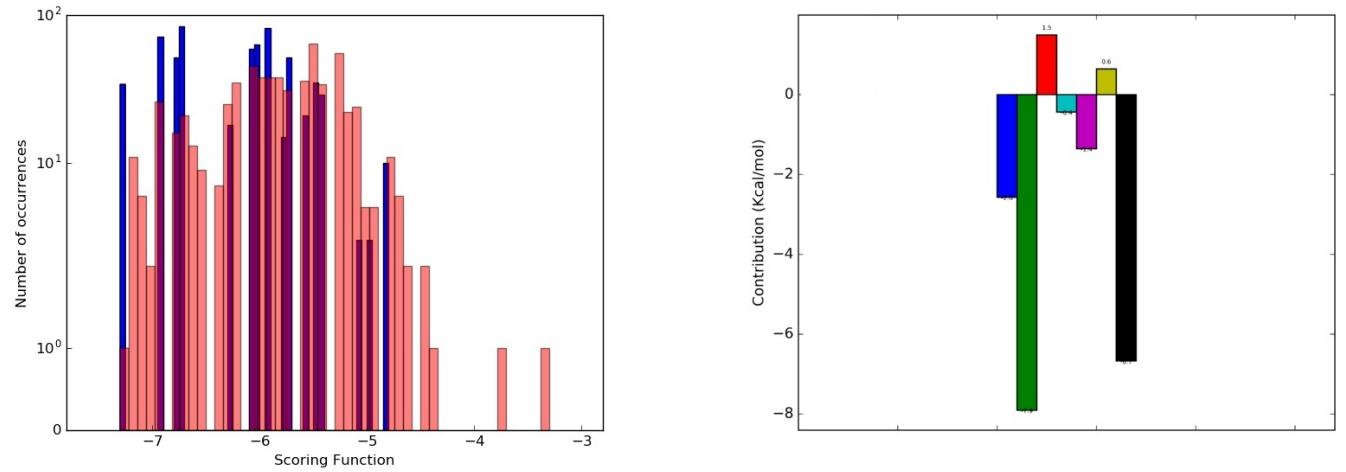

Figure 2: Left panel: Histogram of the distribution of values of the scoring function obtained after BD simulations for each of the three compounds over IL-18. Right panel: Energetic analysis of the top pose from each compound docked to IL-18. Representation of the values of the different energetic contributions to the predicted binding energy $(\mathrm{kcal} / \mathrm{mol})$ where depicted energetic contributions are; electrostatic interaction types Gauss1 and Gauss2 (blue and green color), repulsion forces (red color), hydrophobic interactions (light blue), hydrogen bonds (pink color), entropic contribution (yellow color) and total predicted binding energy (black). Paclitaxel, vincristine and vinorelbine are shown at top, middle and bottom, respectively. 

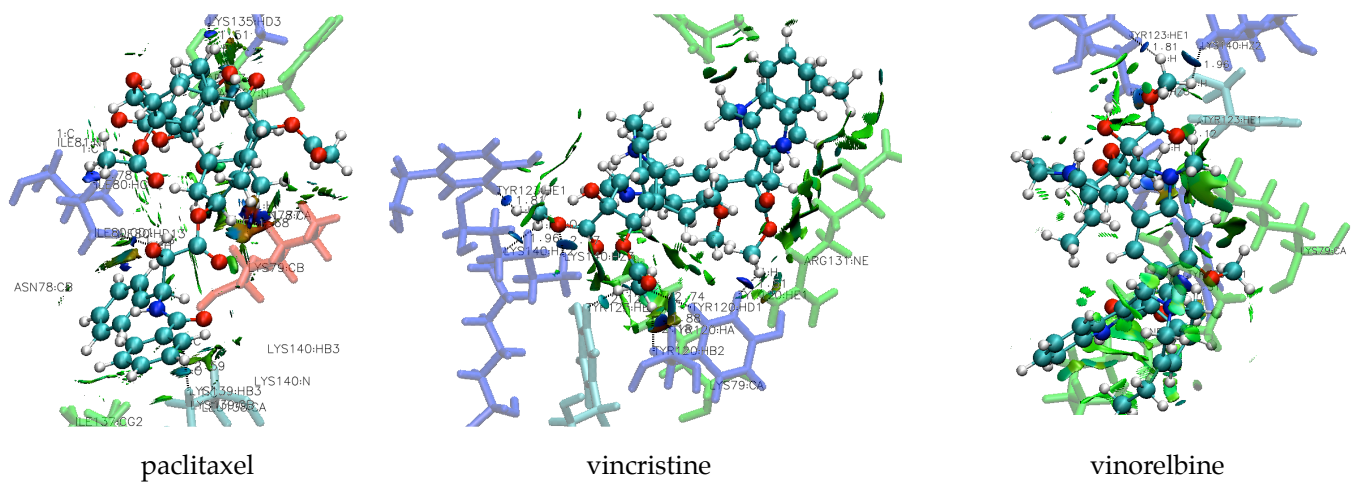

Figure 3: NCI analysis of main alkaloids-IL-18 residue non-covalent interactions in the binding pocket. Color scheme for paclitaxel binding site (left panel): D:Ile80 and D:Lys135 in blue; D:Arg27 and D:Ile137 in green, B:Lys79 in red. Vincristine binding site (middle panel): B:Lys79, D:Ile80 and D:Asn78 in blue; D:Lys135, D:Arg131, D:Ile137 in green. Vinorelbine binding site (right panel): B:Tyr123, B:Lis140, B:Tyr120 in blue, D:Arg131, D:Asp132 and B:Lys79 in green. Isosurfaces color code: the H-bonds are shown as blue surfaces and weak attractive interactions are given as green regions (threshold of $\mathrm{s}=0.5$ au).

cristine and IL-18. In this case, dispersion is much more favored compared to the results obtained for paclitaxel. More specifically, vincristine is very complementary along Arg131. In addition, the ligand is anchored through an important number of hydrogen bonds (blue surfaces) on another part of the pocket, which ensures a large interaction energy. Finally, NCI analysis shows similar result to vinorelbine, which forms H-bonds though B:Tyr123 and B:Lis140 residues and a stable complementary along the benzenic core of the drug with D:Arg131. The hole created around the central pyridinic group is also complemented by Lis79 in an optimal way.

As noted above, docked structures might produce steric interactions with embedded drugs since the protein is not allowed to relax. Following the proposed methodology, MD simulations have been next performed to further refine "raw" docking structures through a full relaxation of the system. The combination of docking and MD approaches accounts for both drug and protein flexibility to mimic the induced-fit effects in the structure of the binding site [34]. Accordingly, the three top BD poses (Figure 1) are equilibrated in water boxes and subjected to MD simulations during trajectories of $25 \mathrm{~ns}$. Figure 4 shows all-heavy atoms root-mean-square displacement (RMSD) of 


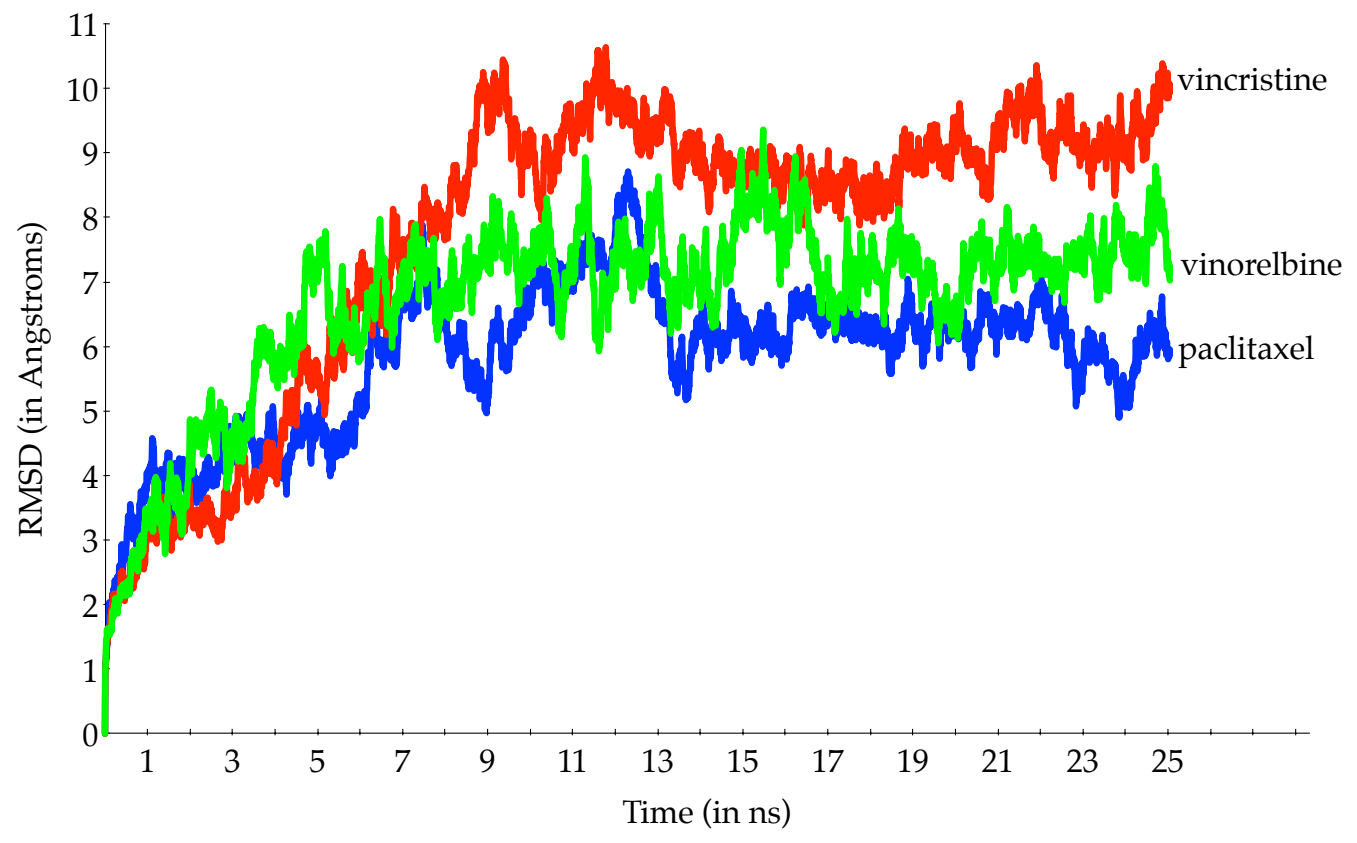

Figure 4: Time dependence of all-heavy atoms RMSD (in $\AA$ ) of IL-18 in the presence of the selected alkaloid-based anticancer drugs during MD trajectories of 25 ns. Blue, red and green lines stand for paclitaxel, vincristine and vinorelbine molecules, respectively. 
the host relative to its initial structure vs. time for selected alkaloids. All MD simulations lead to a similar conclusion: the three selected alkaloid-based anticancer drugs remain within the central cavity of IL-18, which hints that the formed non-covalent interaction pattern is strong enough to stabilize the cargo inside the host structure. A closer inspection reveals that three cargohost systems reach the equilibrium after $15 \mathrm{~ns}$, with a RMSD saturation of ca. $1 \AA$. Consequenlty, IL-18 interactions with the alkaloids were monitored throughout the last $10 \mathrm{~ns}$ of simulation $(15-25 \mathrm{~ns})$ to obtain deeper insights into the dynamic behavior of the binding sites.

As one can see in Figure 5, most of the interactions identified by NCI in the docked structure are retained during MD simulations. According to the produced MD trajectory, paclitaxel binding mode is dominated by H-bonds interaction pattern with a large number of bonds with residues B:Asn78, B:Lys140, D:Ile81, D:Lys135 and D:Leu138. It is worth noting that the clash of paclitaxel with B:Lys79 disappears during the trajectory. Indeed, B:Lys79 significantly contributes to the stabilization of the binding site through a water bridge. This interaction cannot reproduced by our initial docking simulations as they do not include explicit water molecules. This finding confirms the MD refinement as a key step in the computational protocol to properly describe the loading process in the synthesis of novel host-cargo systems.

\section{Conclusions and outlook}

Herein, we performed docking, non-covalent analysis and molecular dynamics simulations to assess the possible use of IL-18 as carrier molecule for anticancer drugs. According to our predictions, IL-18 efficiently embeds three alkaloids with anticancer activity, e.g., paclitaxel, vincristine and vinorelbine, in its central cavity, so that the biological activity of the cytokine is preserved while allowing the selective transport of the embedded drugs.

Of course, a larger panel of anticancer drugs will be required to define the most efficient anticancer drug that can be loaded into the structure of IL18 cytokine (i.e., by including metallodrugs, anthracyclines, antimetabolites, etc). Simultaneously to perform such theoretical calculations, the cytokine as carrier molecule can be already tested in vivo to validate their future clinical applications. 

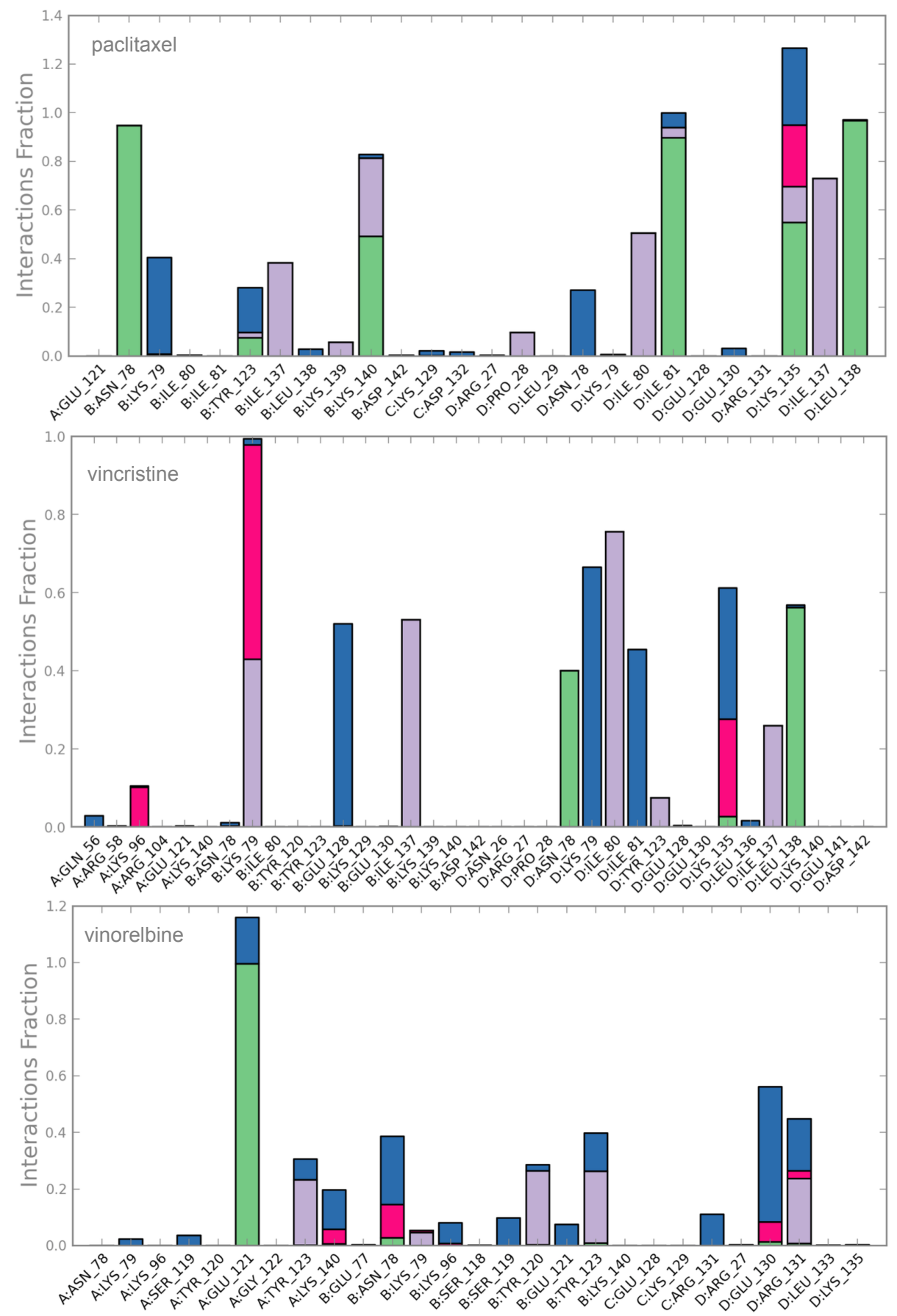

Figure 5: Decomposition of main protein-ligand interactions for IL-18 embedded alkaloids throughout the last $10 \mathrm{~ns}$ of MD simulations. Color code: H-bonds in green, dispersion interactions in pink, ionic contacts in pink and water bridges contribution in blue. 


\section{Acknowledgements}

This work was partially supported by Fundación SénecaAgencia de Ciencia y Tecnología de la Región de Murcia under Projects 19419/PI/14-1 and 18946/JLI/13. J.P. C.-C. acknowledges the support provided by the Centro de Alto Rendimiento de la Regin de Murcia within its Research Program (CFE-CAR-23/15). This research used resources of the Plataforma Andaluza de Bioinformtica installed at the Universidad of Málaga, the supercomputing infrastructure of Poznan Supercomputing Center, and the local Galileo cluster installed at UCAM.

\section{References}

[1] G. M. Cragg, D. J. Newman, Plants as a source of anti-cancer agents, J. Ethnopharmacol. 100 (2005) $72-79$.

[2] R. L. Noble, C. T. Beer, J. H. Cutts, Role of chance observations in chemotherapy: Vinca rosea, Ann. N. Y. Acad. Sci. 76 (1958) 882-894.

[3] R. van der Heijden, D. I. Jacobs, W. Snoeijer, D. Hallard, R. Verpoorte, The catharanthus alkaloids: Pharmacognosy and biotechnology, Curr. Med. Chem. 11 (2004) 607-628.

[4] E. Rowinsky, Holland-Frei Cancer Medicine, Hamilton (ON): BC Decker, 6th edition edition.

[5] R. H. Himes, Interactions of the catharanthus (vinca) alkaloids with tubulin and microtubules., Pharmacol. Ther. 51 (1991) 257-267.

[6] E. Blanco, H. Shen, M. Ferrari, principles of nanoparticle design for overcoming biological barriers to drug delivery, Nat. Biotechnol. 33 (2015) 941-951.

[7] C. Sanchez-Cano, M. J. Hannon, Novel and emerging approaches for the delivery of metallo-drugs, Dalton Trans. (2009) 10702-10711.

[8] A. Yadav, S. Sharma, V. K. Yadav, Non-covalent carriage of anticancer agents by humanized antibody trastuzumab, J. Mol. Model. 22 (2016) $112-126$. 
[9] H.-S. Cho, K. Mason, K. X. Ramyar, A. M. Stanley, S. B. Gabelli, D. W. Denney, Jr, D. J. Leahy, Structure of the extracellular region of her2 alone and in complex with the herceptin fab, Nature 421 (2003) 756-760.

[10] C. A. Hudis, Trastuzumab - mechanism of action and use in clinical practice, N. Engl. J. Med. 357 (2007) 39-51.

[11] J. Gao, Y. G. Liu, R. Liu, R. Zingaro, Herceptin-platinum(ii) binding complexes: Novel cancer-cell-specific agents, ChemMedChem 3 (2008) 954-962.

[12] C. Xu, B. Wang, S. Sun, Dumbbell-like aufe3o4 nanoparticles for targetspecific platin delivery, J. Am. Chem. Soc. 131 (2009) 4216-4217.

[13] J. P. Cerón-Carrasco, J. Cerezo, A. Requena, J. Zúñiga, J. ContrerasGarcía, S. Chavan, M. Manrubia-Cobo, H. E. Pérez-Sánchez, Labelling herceptin with a novel oxaliplatin derivative: a computational approach towards the selective drug delivery, J. Mol. Model. 20 (2014) 2401-2409.

[14] R. V. J. Chari, M. L. Miller, W. C. Widdison, Antibody-drug conjugates: An emerging concept in cancer therapy, Angew. Chem. Int. Ed. 53 (2014) 3796-3827.

[15] Z. Ma, W. Li, S. Yoshiya, Y. Xu, M. Hata, Y. El-Darawish, T. Markova, K. Yamanishi, H. Yamanishi, H. Tahara, Y. Tanaka, H. Okamura, Augmentation of immune checkpoint cancer immunotherapy with il18, Clin. Cancer Res. 22 (2016) 2969-2980.

[16] J. L. Sussman, D. Lin, J. Jiang, N. O. Manning, J. Prilusky, O. Ritter, E. Abola, Protein data bank (pdb): database of three-dimensional structural information of biological macromolecules, Acta Crystallographica Section D: Biological Crystallography 54 (1998) 1078-1084.

[17] N. Tsutsumi, T. Kimura, K. Arita, M. Ariyoshi, H. Ohnishi, T. Yamamoto, X. Zuo, K. Maenaka, E. Y. Park, N. Kondo, M. Shirakawa, H. Tochio, Z. Kato, The structural basis for receptor recognition of human interleukin-18, Nat. Commun. 5 (2014).

[18] G. Madhavi Sastry, M. Adzhigirey, T. Day, R. Annabhimoju, W. Sherman, Protein and ligand preparation: parameters, protocols, and influence on virtual screening enrichments, J. Chem. Inf. Model. 27 (2013) $221-234$. 
[19] H. Li, A. D. Robertson, J. H. Jensen, Very fast empirical prediction and rationalization of protein pka values, Proteins 61 (2005) 740-721.

[20] I. Sánchez-Linares, H. Pérez-Sánchez, J. M. Cecilia, J. M. García, Highthroughput parallel blind virtual screening using bindsurf, BMC bioinformatics 13 (2012) 1.

[21] J. Navarro-Fernández, H. Pérez-Sánchez, I. Martínez-Martínez, I. Meliciani, J. Guerrero, V. Vicente, J. Corral, W. Wenzel, In silico discovery of a compound with nanomolar affinity to antithrombin causing partial activation and increased heparin affinity, Journal of medicinal chemistry 55 (2012) 6403-6412.

[22] O. Trott, A. J. Olson, Autodock vina: improving the speed and accuracy of docking with a new scoring function, efficient optimization, and multithreading, Journal of computational chemistry 31 (2010) 455-461.

[23] J. Contreras-García, E. R. Johnson, S. Keinan, R. Chaudret, J. P. Piquemal, D. N. Beratan, W. Yang, Nciplot: A program for plotting noncovalent interaction regions, J. Chem. Theory Comput. 7 (2011) 625-632.

[24] E. R. Johnson, S. Keinan, P. Mori-Sánchez, J. Contreras-García, A. J. Cohen, W. Yang, Revealing noncovalent interactions, J. Am. Chem. Soc. 132 (2010) 6498-6506.

[25] J. Contreras-García, W. Yang, E. R. Johnson, Analysis of hydrogenbond interaction potentials from the electron density: Integration of noncovalent interaction region, J. Phys. Chem. A 115 (2011) 1298312990 .

[26] H. J. C. Berendsen, J. P. M. Postma, W. F. van Gunsteren, J. Hermans, Intermolecular Forces, Reidel, Dordrecht, pp. 331-342.

[27] S. Nosé, A molecular dynamics method for simulations in the canonical ensemble, Mol. Phys. 52 (1984) 255-268.

[28] W. G. Hoover, Canonical dynamics: equilibrium phase-space distributions., Phys. Rev. A 31 (1985) 1695-1697.

[29] G. J. Martyna, D. J. Tobias, M. L. Klein, Constant pressure molecular dynamics algorithms, J. Chem. Phys. 101 (1994) 4177-4189. 
[30] M. Tuckerman, B. J. Berne, G. J. Martyna, Reversible multiple time scale molecular dynamics, J. Chem. Phys. 97 (1992) 1990-2001.

[31] T. A. Darden, D. M. York, L. G. Pedersen, Particle mesh ewald: An $\mathrm{n}-\log (\mathrm{n})$ method for ewald sums in large systems, J. Chem. Phys. 98 (1993) 10089-10092.

[32] Desmond molecular dynamics system, version 4.4, D. E. Shaw Research, New York, NY. Maestro-Desmond Interoperability Tools, version 4.4, Schrödinger, New York, NY, 2015.

[33] D. B. Kitchen, H. Decornez, J. R. Furr, J. Bajorath, Docking and scoring in virtual screening for drug discovery: methods and applications, Nat Rev Drug Discov 3 (2004) 935-949.

[34] H. Alonso, A. A. Bliznyuk, J. E. Gready, Combining docking and molecular dynamic simulations in drug design, Med. Res. Rev. 26 (2006) $531-568$. 